\title{
Smart Health Management Technology
}

\author{
Hiroshi Nakajima
}

Omron Corporation

Japan

\section{Introduction}

The notion of health management technology (HMT) is simple but powerful because it employs the ideas of cyclical evolution and synergetic integration of devices and services based on causality and human machine collaboration (Nakajima, 2008a). It can be applied for the different entities of human beings, artifacts, and nature environment as following discussions.

An essential and simple observation and understanding of our world reveals that it can be considered as comprising humans, artifacts, and nature environments shown in Fig.1. Even though the values of the entities are given by humans, they have obviously different directions. Examples of such values are comfort and safety for humans, efficiency and effectiveness for artifacts, and environmental enhancement for nature. The problem is that these values come into conflict with each other. Examples of conflicts in a factory are as follows. Productivity related to efficiency and effectiveness is the most important value in manufacturing lines. However, the focus only on productivity will increase the emission of carbon dioxide and other contaminants that will negatively influence to the sustainability of nature environment, and the safety and comfort of human operators in the manufacturing line. Thus, the conflicts of values among the respective entities cause serious problems in important areas such as the environment, agriculture and food, security and safety, and human health these days. In this sense, harmonization among them should be realized with keeping good health condition of each entity for realizing a desired next society. Although this vision might look grandiloquent, health management of each entity is considered as important activity as steady steps toward the bright future.

Because of recent development of information and communication technology (ICT), sensory networks have been pervading various fields such as home security, healthcare, condition-based maintenance for manufacturing equipment, and environment monitoring. They require the suitable integration of both sensing devices and valuable services.

HMT is designed for providing basic four kinds of functions by centering causality. The functions are measurement, recognition, estimation, and evolution. The functions provide the solution of cyclical evolvement based on causality which abstractly illustrates conditions of target systems and is used as problem solving knowledge, which is composed of feature attributes extracted from sensory data and intermediate characteristics. In this sense, causality could evolve and be updated according to sophistication of sensing and control mechanisms. Because the nature of causality is transparent to humans, the structure can be easily improved through human-machine collaboration. This feature of the technology is quite important because the integration of human knowledge and sensory data will bring a 
powerful and sophisticated solution for complex problems. HMT has been applied to various types of applications such as human healthcare, machine health monitoring in manufacturing process, and energy management systems. Some case studies of human health care are introduced in the article.

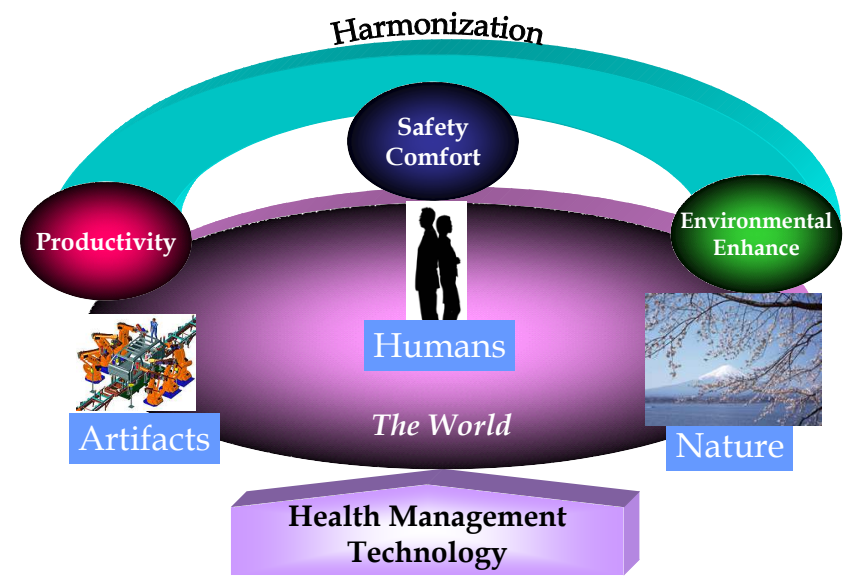

Fig. 1. The world consists of humans, artifacts, and nature environment

The rest of this chapter is organized as follows; Section 2 proposes Health Management Technology; Section 3 and 4 introduce the applications of visceral fat estimation and heart rate estimation respectively; Section 5 concludes this chapter.

\section{Health management technology}

In this section, the notion of the health management technology (HMT) is introduced to discuss its basic mechanisms and the four functions defined in the technology framework. It employs causality as an essential solution component in the technology. Fig.2 shows the structure of the target systems and HMT.

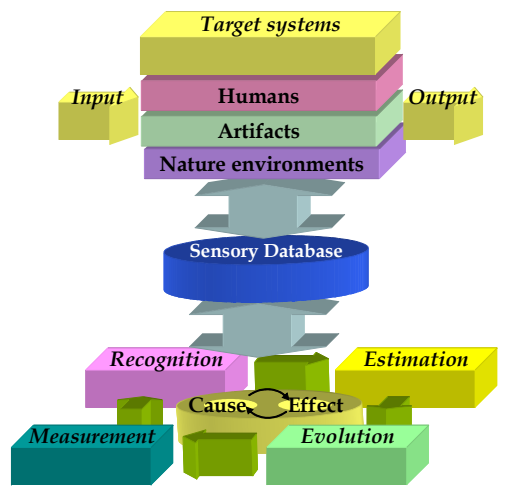

Fig. 2. Structure of the target systems and health management technology centering sensory database and causality 


\subsection{Overviews}

Fig.3 shows an overview of HMT based on causality. The objective of the technology is to estimate the health condition of humans, artifacts, and nature to improve their health. Because the target system continuously changes and their health management systems must adapt to these changes, the cause-effect structure must evolve cyclically and continuously according to sophistication of both the target system and its management side. In HMT, four functions are defined for cyclically evolving the model as shown in Fig.3.

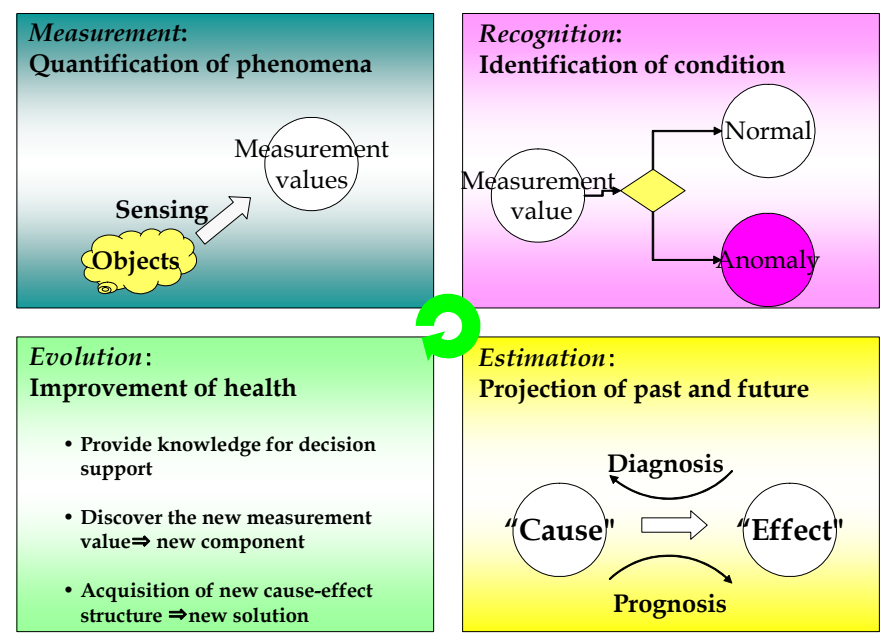

Fig. 3. Four functions of health management technology

- Measurement is to quantify of phenomena to arrive at a value from analyzing signals from sensors. The function is realized by the elemental technologies of feature extraction, feature selection, and feature evaluation.

- Recognition is to identify the condition of the target system using the measured value. The function is based on the pattern recognition technologies such as discrimination, classification, and identification.

- Estimation is to project the past and future status of the system. The functions of diagnosis and prognosis are realized by employing cause-effect structure. The elements of technology for realizing this function are probability graphs and causal models such as Bayesian network, structure equation model, etc.

- Evolution is to improve the target system and to update causality by the discovery of new events and make changes in the target system. The function should be realized by human-machine collaborative systems analysis and design.

It is important for us to manage our health by considering diet meal, sleep and rest, and exercise. There is important causality among these lifestyle habits and vital signals such as blood pressure, blood glucose, and blood adipose. Even though it has not been realized yet, the ideal example is shown in Fig.4.

The example of human health management is introduced for explaining effectiveness and efficiency of multivariate time series data and their cause-effect structure. The sensory data are used for composing the causality that can be applied to prevent diseases and to improve health. Among biological information, blood pressure is usually used as an important index 
of health condition because it is closely associated with cardiovascular events such as brain infarction, stroke, myocardial infarction, and heart failure. Besides other important factors such as total cholesterol, casual glucose, etc., blood pressure is easy to be measured at home and medical facilities. There are also easy and useful indices; body composition, active mass, and sleep condition. The composition of muscle and fat is measured by a body composition monitor. The indices could be used as one of alternative reference indices for quality and quantity of diet in our life. An active mass monitor or a pedometer could measure types, intensity, and quantity of exercises in daily life. A sleep monitor measures quality and quantity of sleep. The multivariate time series data of blood pressure, weight or body composition of muscle and fat, steps or active mass, and quality and quantity of sleep could be gathered by the sensing devices. The cause-effect structure derived from the data will provide important knowledge for diagnosis and prognosis of health condition optimized for individuals. For instance, continuous and well active mass seems to have good influence on the body composition and sleep to realize the stability of blood pressure.

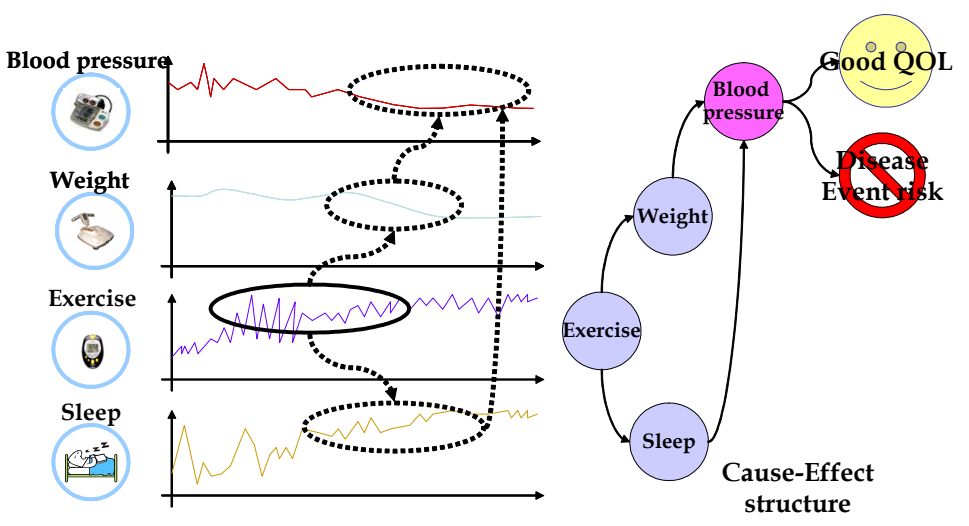

Fig. 4. Multivariate time series data and causal analysis for life style and vital signal

\subsection{Discussions on invasion, intrusion, and consciousness}

As long as the application domain in human health management, it is required for us to discuss and study on the relationship between human live body and instrumentation. In this section, invasion, intrusion, and consciousness in biomedical sensing are studied and discussed from the views of both human burden and technology performance. Invasion has been studied more so far than intrusion and consciousness. However, latter two issues are also important to provide different meanings and values from invasion.

\subsubsection{Invasion}

Invasion levels can be defined by distance between sensory head and sensing target or location of them (Yamagoe, 2000). There are five invasion level of biomedical sensing as follows;

1. A catheter is inserted into body to send transmitter to a sensor head.

2. A sensor head is implanted to send sensory signals via fixed line.

3. A sensor head is implanted to send sensory signals via wireless communication.

4. A sensor head is contacted on skin of the body to detect signals.

5. A sensor head is not contacted on skin of the body to detect signals. 
As mentioned above, the invasion levels are defined by the positional relationship between sensory head and live body. Compared with invasion, intrusion and consciousness sensing are new ideas in biomedical but important for both of sensing accuracy and human comfort.

\subsubsection{Intrusion}

Nonintrusion is important idea for sensing daily life activity. Electrocardiogram (ECG) and blood pressure (BP) are very important physical indices used in medical and healthcare. The indices can be measured in just in hospital and clinic. It is strongly required to monitor for 24 hours to detect anomaly condition of body in daily life. However, it is impossible to monitor ECG in daily life in the case of using ordinary ECG equipment. A Holter electrocardiographic monitor has been realized for gathering the ECG signals and an ambulatory blood pressure monitor for accumulating blood pressure signals for 24 hours. Although the equipment could be used for sensing live body, it is far from non-intrusion. The user has to use contacted sensory head and to carry on the equipment.

\subsubsection{Consciousness}

Consciousness or realization to be sensed would have some influence to the sensing signals. White coat hypertension and masked hypertension (Messerli, 2005) are serious examples for understating the importance of unconsciousness sensing. Not just for these serious cases, other examples can be easily pointed out with considering consciousness such as video and image sensing. Long and continuous sensing for 24 hours requires unconsciousness. Besides them, unconsciousness helps for users not to forget the measurement.

Through these definitions and observations on invasion, intrusion, and unconsciousness, some discussions on influences to patients and live bodies and to measurements.

\subsubsection{Consideration on humans and instrumentation ends}

It is strong requirements to reduce and to eliminate burden, pain, and damage both physically and mentally to live bodies and patients. According to the definitions of invasion level, the level is decided by distance and location between sensor head and body. However, even though X-ray CT scan is non contact sensing, it gives serious impact of X-ray exposure on the live body. Implant sensing requires surgery whose invasion level is quite high, but intrusion is very low when the sensor could be used for a long time. Additionally, a patient who is implanted sensor into could feel unconscious with the sensor for long time usage. As these discussions, invasion is not the only perfect idea in biomedical sensing. Intrusion and consciousness should be also thought for realizing the effective and efficient bioinstrumentation.

Sensing performance would be generally good with high invasion level. However, invasion sometimes brings negative results in sensing. Contact with sensor head and live body each other makes energy exchange between them to cause instability in sensing. For example, huge sensor head of clinical thermometer realizes good fitting with body however it takes body temperature. Like the examples pointed out in intrusion section, it would be very hard to keep the ideal situation of the patient such as in daily life activities with high intrusion. Besides, unconscious sensing might bring good results with the sensing performance as discussed in consciousness section.

Realization of biomedical sensing without invasion, intrusion, and consciousness brings some uncertainty in the sensing mechanism. Sensor signals superimpose not just target but 
other kinds of signals. Besides extraction of target signal, it is also required to compose causal structures which explain sensing principles well.

\subsection{Causal analysis}

Causal analysis studies have covered wide and various areas from psychology and philosophy (Pearl, 2000; Gopnik 2007) to image processing and bioinformatics (Mittal, 2007). Theoretical basics were also deeply studied (Whittaker, 1990) and some studies covered wide areas (Pearl, 2000; Lucas, 2007; Spirtes 2000). In this article, the path analysis that is one of principle causal analysis methods is employed to realize HMT as a first step of the technology because of its simple but powerful solution nature. Besides introduction to the method, acquisition and improvement of cause-effect structure are discussed.

As one of cause-structure acquisition, the path analysis (Wright, 1923) or the structural equation model (Scheines, 1999) is introduced here. The cause-effect structure is described by a cause-effect diagram shown in Fig.4.

The cause-effect diagram is formulated by the structural equations (1)-(3). A causal relationship between variables is quantified by the coefficients $\alpha_{Y Z}$ which are called causal effect or path coefficient. The paths from $X_{1}$ to $X_{4}$ are the direct effect of $X_{1} \rightarrow X_{4}$, the indirect effects of $X_{1} \rightarrow X_{2} \rightarrow X_{4}$ and $X_{1} \rightarrow X_{2} \rightarrow X_{3} \rightarrow X_{4}$. The sum of the direct and indirect effects is called total effect which is calculated by the equation (4). By using these effects, the cause-effect structure can be quantified. Sometimes for simplification, means and variances of variables used in the cause-effect structure are normalized to 0 and 1 respectively.

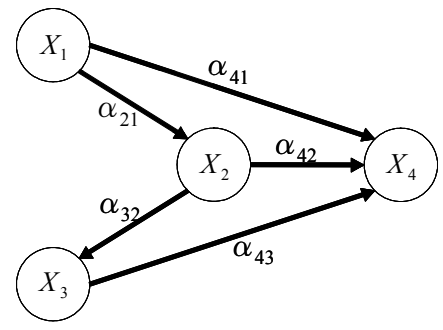

Fig. 5. Causality example

$$
\begin{gathered}
X_{2}=\alpha_{21} X_{1}+\varepsilon_{1} \\
X_{3}=\alpha_{32} X_{2}+\varepsilon_{2} \\
X_{4}=\alpha_{41} X_{1}+\alpha_{42} X_{2}+\alpha_{43} X_{3}+\varepsilon_{3} \\
E_{T 41}=\alpha_{41}+\alpha_{21} \alpha_{42}+\alpha_{21} \alpha_{32} \alpha_{43}+\varepsilon_{4}
\end{gathered}
$$

Besides model representations, causality acquisition methods have been studied. A Bayesian approach can be applied when data are categorical (Heckerman, 1999). Regarding sensing data, which are continuous values, a causality acquisition method using the data is studied for applying anomaly detection of a discrete manufacturing process (Endo, 2008). One of the reasons why cause-effect structure is employed as performable knowledge is its transparent 
nature for human machine collaboration. That is to say, the transparent aspect of causality would bring a more powerful solution to human machine collaborative improvement (Marutschke, 2008, 2009).

While a cause-effect structure has been acquired, progressive improvement of the structure is required according to the change of target systems. There are different directions of causality improvement. One is simplification or abstraction and the other complication or concretion. These ideas are useful to reduce the cost and to improve accuracy of health management. For instance, in the case of multiple sensors required for health management such as lifestyle habit monitoring which considers exercise, diet, and sleep, reduction of the number of sensors through updating cause-effect structure will work well for cost reduction of sensors and data management. On the other side, complication of causality would work for increasing estimation accuracy of target systems' condition. For adapting to the increase of system's complexity and preciseness, transformation of cause-effect structure is discussed from the viewpoint of a hierarchical modeling method (Tsuchiya, 2008, 2010).

\section{Visceral fat estimation}

In the medical profession, it has been realized that visceral fat (VF) is main cause of lifestyle diseases and metabolic syndrome. According to the trend, a medical instrument of VF measurement has been strongly required with low invasion, low cost, and ease of use. In this section, a visceral fat estimation method by bioelectrical impedance and causal analysis is proposed to realize the practical device with low invasion and low intrusion in the medical fields.

Metabolic syndrome is not just obesity disease but is associated with serious diseases such as diabetes mellitus and hypertension. They would cause complicating illness from diabetes and cardiovascular events to decrease quality of life. Even though the regulations in the countries might be slightly different, the indices of blood pressure, blood lipid, blood glucose, and visceral fat are mainly used for the criterion of medical diagnosis. First three criteria can be measured by a blood pressure meter and blood investigation with high accuracy. However, because VF of live body can not be measured directly, some other indices are generally used, such as body mass index, waist-hip ratio, and waist circumference. However, these indices are not accurate to estimate visceral fat volume. On the other hand, the cross sectional area at umbilicus level by using an X-ray computerized tomography (CT) scan or a magnetic resonance imaging (MRI) is the gold standard in medical fields for measurement of visceral adipose tissue (ECCODJ, 2002; Gomi, 2005). However, a CT scan causes X-ray exposure and requires difficulty of use and high cost. For solving these problems, a medical instrument is strongly required with non invasion, low intrusion, ease of use, and low cost. Besides these benefits, the instrument without X-ray exposure can be used for the follow-up measurement of visceral fat reduction after medical treatment for metabolic syndrome and obesity.

In response to the requirements, the visceral fat estimation method has been proposed by employing bioelectrical impedance analysis and causal analysis for realizing the medical device of VF measurement. Two kinds of impedance and information of body shape at umbilicus level are used as sensory data. The causal structure is designed by considering the measurement principle to be optimized based on statistical analysis to estimate visceral fat area as the dependent variable which is provided by a CT scan and image processing. The experiments were conducted to investigate the performance of the proposed method. The 
result was 0.88 coefficient of correlation value between the proposed instrument and a CT scan. The method works well to realize the practical instrument used in medical field. Besides the performance, the model brings understandability and transparency with the measurement nature of VF.

\subsection{Analysis and design methods}

In this section, the measurement principles are introduced and the related works are surveyed. Bioelectrical impedance analysis (BIA) and causal analysis (CA) follows the principles.

\subsubsection{Measurement principles}

Fig. 6 shows a cross sectional area of the human body at umbilicus level provided by a CT scan and colored by image processing. There are three types of composition which are visceral fat, subcutaneous fat, and lean body. Lean body is not fat and internal organs, muscles, and bones. Each area can be calculated automatically by image processing technology applied to the image of cross sectional area gotten by a CT or an MRI. Visceral fat area can be calculated by the equation (5). It is the measurement principle of VF.

$$
V F A=C S A-S F A-L B A
$$

where $V F A$ is visceral fat area, CSA all cross sectional area, SFA subcutaneous area, and LBA lean body area.

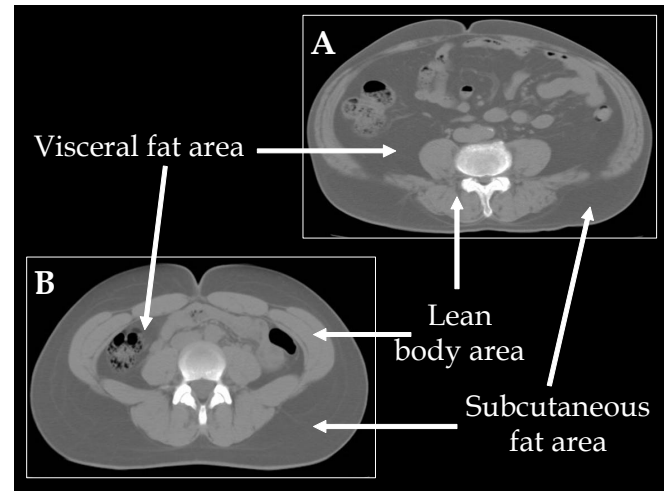

Fig. 6. Cross sectional area image by CT scan and Image Processing

Related studies to estimate visceral fat area have been done. All studies introduced here employ BIA because it is commonly and practically used for estimating body composition. Ryo et al. proposed the method which employs waist circumference and abdominal impedance which occurs at the flank to the flow of current between the umbilicus and the back (Ryo, 2005). They are used as the feature attributes in the equation (6).

$$
\tilde{A}_{V F 1}=\alpha_{1} V o W c^{3}+\varepsilon
$$

where $\tilde{A}_{V F 1}$ is the estimated $V F A, V o$ the impedance measured at the flank, $W_{c}$ waist circumference, $\alpha_{1}$ and $\varepsilon$ regression coefficient and error term respectively. 
Shiga et al. proposed the model to estimate VFA, which employed two types of impedance and will be described in Section 3.1.2. The model is given by the equation (7) (Shiga, 2007, 2009).

$$
\tilde{A}_{V F 2}=\alpha_{1} W c^{2}+\alpha_{2} W c Z_{S}+\alpha_{3} / Z_{T}+\varepsilon
$$

where $\tilde{A}_{V F 2}$ is the estimated VFA, $Z_{S}$ the surface abdominal impedance, $Z_{T}$ the total abdominal impedance, $\alpha_{i}(i=1,2,3)$ and $\varepsilon$ regression coefficient and error term respectively. The model uses the only measured variables of waist circumference and the impedances. Some studies proposed the several models using the width and height of the cross sectional area of an abdomin (Shiga, 2009; Yoneda, 2007, 2008). Some of them also employ other variables such as gender and age besides them (Yoneda, 2007, 2008).

One of the models is given by the equation (8) which considers influence by gender and age.

$$
\begin{aligned}
\tilde{A}_{V F 3} & =\alpha_{1} a+\alpha_{2} b+\alpha_{3} / b+\alpha_{4} a^{2}+\alpha_{5} b^{2} \\
& +\alpha_{6} / b^{2}+\alpha_{7} / Z_{T}+\alpha_{8} Z_{S} \sqrt{a^{2}+b^{2}} \\
& +\alpha_{9} A+\alpha_{10} G+\varepsilon
\end{aligned}
$$

where $a$ and $b$ are the width and the height of a cross sectional area shown in Figure $2, Z_{S}$ the surface abdominal impedance, $Z_{T}$ total abdominal impedance, $A$ age, $G$ gender, and $\alpha_{i}(i=1,2, \ldots, 10)$ and $\varepsilon$ regression coefficient and error term respectively.

The model denoted by the equation (7) is the most similar to the measurement principle given by the equation (5). However, the information of abdominal shape might be eliminated by using waist circumference. On the other side, height and width of the cross sectional area of abdomin would bring some information about it. The variables improved the estimation performance (Tsuchiya, 2010) but age and gender are not suitable for estimating VFA because they are not physical variables and not shown in the measurement principle given by the equation (5). The equation (8) seems to cause some problems of complexity and multicollinearity because it employed many variables and multiple appearances in different terms.

\subsubsection{Bioelectrical impedance analysis}

In order to extract visceral fat from the all composition of abdominal human body, two types of bioelectrical impedances named Dual Impedance (DI) are employed as shown in Fig.7. In the DI method, the electrodes are placed on the both hands' backs and the both feet's insteps with the subjects lying on the back. The surface abdominal impedance $Z_{S}$ is measured by using the electrodes at the back. The electric currents are passed among the electrodes on the back. The total abdominal impedance $Z_{T}$ is measured by using the electrodes placed on hands, feet, and abdomin. The currents are passed from both hands and feet to abdomin.

The surface abdominal impedance is highly correlated with the subcutaneous fat volume (Scharfetter, 2001) and the total one is inversely correlated with the lean body volume; that is fat-free mass (Lukaski, 1985). Thus, our strategy is to develop the estimation model which could explain well the measurement principle as shown in the equation (5) by using the DI method. 


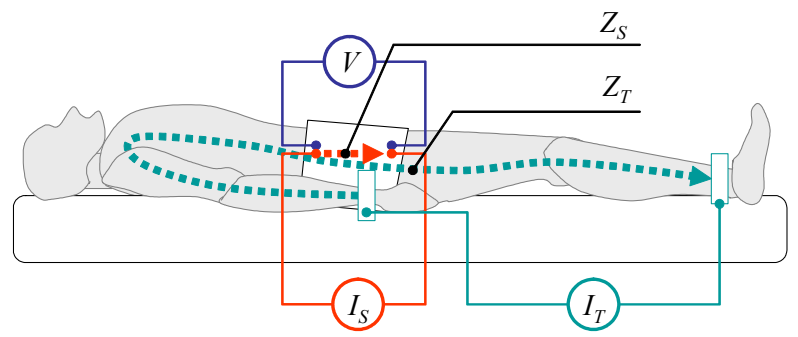

Fig. 7. Human body and the two types of impedances

\subsubsection{Causal analysis}

Fig.8 illustrates the causality of VFA estimation considering the relationship between the sensory variables and the measurement principle. The left and the right are the sensory variables and the principle measurement respectively. According to the causal structure, we prepare the variables which are the candidates for constructing the causality. By using the selected variables, multi linear regression was employed to acquire the estimation model. In this step, we carefully considered about nature of the model without over-fitting, multicollinearity, and some other difficulties; especially keeping the notion of the measurement principle. We finally derived the estimation model denoted by the equation (9).

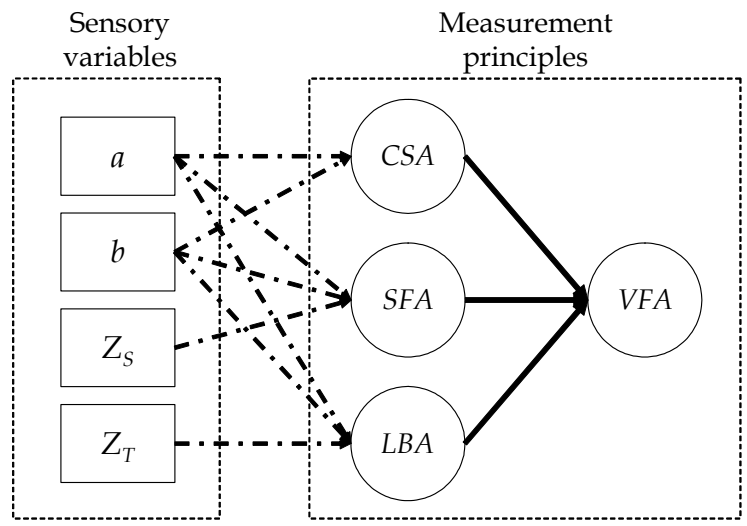

Fig. 8. Causality between the sensory variables and the measurement principle

$$
\tilde{A}_{V F 4}=\alpha_{1} a+\alpha_{2} b^{2}+\alpha_{3} Z_{S} \sqrt{a^{2}+b^{2}}+\alpha_{4} / Z_{T}+\varepsilon
$$

In order to extract the causality for estimating VFA, we designed the following development steps;

1. Preparation of variables according to the measurement principles

2. Selection of the variables by applying AIC and VIF on the sample dataset

In the first step, we prepared the candidates of the variables as possible as used in the estimation equation. The idea here is that the candidates should bring some information about VFA estimation and be understandable from the point view of the measurement principles shown in the figure 8 . The prepared variables are listed in Table 1. 


\begin{tabular}{|c|c|c|}
\hline Relations & Variables & Remarks \\
\hline \multirow{4}{*}{$\operatorname{CSA}$} & $a, b$ & $\begin{array}{l}\text { Primary measurement variables. } a \text { and } b \text { are } \\
\text { width and height of a cross sectional area at } \\
\text { umbilicus level as shown in Figure } 2 \text {. }\end{array}$ \\
\hline & $a^{2}, b^{2}$ & Extension of measurement values. \\
\hline & $a b$ & Correlation with a cross section. \\
\hline & $\sqrt{a^{2}+b^{2}}$ & Both size and shape information. \\
\hline \multirow[t]{2}{*}{ SFA } & $Z_{S}$ & $\begin{array}{l}\text { A primary measurement variable of } \\
\text { bioelectrical impedance. } Z_{S} \text { is surface } \\
\text { abdominal impedance. It has high } \\
\text { correlation with } S F A \text { (Scharfetter, 2001). }\end{array}$ \\
\hline & $\begin{array}{l}a Z_{S}, b Z_{S}, a^{2} Z_{S}, b^{2} Z_{S} a b Z_{S} \\
\sqrt{a^{2}+b^{2}} Z_{S}\end{array}$ & $\begin{array}{l}\text { The combination of the surface impedance } \\
\text { and the shape and size information of the } \\
\text { cross section. }\end{array}$ \\
\hline \multirow{2}{*}{$L B A$} & $Z_{T}$ & $\begin{array}{l}\text { A primary measurement variable of } \\
\text { bioelectrical impedance. } \\
Z_{T} \text { is total abdominal impedance. It has } \\
\text { high inverse correlation with } L B A \\
\text { (Lukaski, 1985). }\end{array}$ \\
\hline & $\begin{array}{l}a / Z_{T}, b / Z_{T} \\
a^{2} / Z_{T}, b^{2} / Z_{T} \\
a b / Z_{T}, \sqrt{a^{2}+b^{2}} / Z_{T}\end{array}$ & $\begin{array}{l}\text { The combination of inverse of the total } \\
\text { impedance and the shape and size } \\
\text { information of the cross section. }\end{array}$ \\
\hline
\end{tabular}

Table 1. Candidates of the variables used in the estimation equation

The second step is the suitable variables selection as a set of them named tuple. We employed two different criteria to realize the legitimate model for VFA estimation. Our consideration was to realize both performance guarantee and understandability. The first is the accuracy and simplification of the model without over-fitting to the sample dataset. The second is multicollinearity which causes some difficulties in the estimation results. The problem is caused by an almost linear relationship among independent variables in the regression model. Akaike Information Criterion (AIC) (Akaike, 1974) and Variance Inflation Factor (VIF) (Armitage, 2001) are the employed criteria for the former and later considerations. The criteria are given by the equation (10) and by equation (11) respectively.

$$
A I C=n \log \left(2 \pi \hat{\sigma}_{e}^{2}\right)+\frac{1}{\hat{\sigma}^{2}} \sum_{i=1}^{n}\left\{y-\left(\alpha_{1} x_{1}+\cdots+\alpha_{m} x_{m}+\varepsilon\right)\right\}^{2}+2(m+2)
$$

where $m$ and $n$ is the numbers of independent variables and data respectively, $y$ a dependent variable, $x_{i}(i=1,2, \ldots, m)$ independent variables, $\alpha_{i}(i=1,2, \ldots, m)$ coefficient terms, $\varepsilon$ error term, $\hat{\sigma}_{e}$ the standard deviation of estimation errors.

$$
V I F_{i}=1 /\left(1-R^{2}{ }_{i}\right)
$$

where $R_{i}$ is the multiple correlation coefficient between the independent variable $x_{i}(i=1,2, \ldots n)$ and the rest of $(n-1)$ variables. 
The variable sets were constructed by selecting the variables with the combination constraints given by the equations (11).

$$
\begin{aligned}
& X_{i}=\left[x_{j}, x_{k}, x_{l}\right] \\
& \left.x_{j}=\left\{(a, b),\left(a^{2}, b^{2}\right),\left(a, b^{2}\right),\left(a^{2}, b\right), a b, \sqrt{a^{2}+b^{2}}\right)\right\} \\
& x_{k}=\left\{Z_{S}, a Z_{S}, b Z_{S}, a^{2} Z_{S}, b^{2} Z_{S}, a b Z_{S}, \sqrt{a^{2}+b^{2}} Z_{S}\right\} \\
& x_{l}=\left\{1 / Z_{T}, a / Z_{T}, b / Z_{T}, a^{2} / Z_{T}, b^{2} / Z_{T}, a b / Z_{T}, \sqrt{a^{2}+b^{2}} / Z_{T}\right\}
\end{aligned}
$$

where $X_{i}$ is a tuple of independent variables selected from the three categories of CSA, $S F A$, and LBA which consists of $x_{j} \quad(j=1,2, \ldots, 6), x_{k} \quad(k=1,2, \ldots, 7)$, and $x_{l}(l=1,2, \ldots, 7)$. According to the equation (11), there are four or three variable combinations of the candidates. VIF and AIC were applied to all the tuples to select the top 10 according to the regulations of $V I F<10$ and the ascending order of AIC. In this case, VIF values listed in the table are the maximum of all the combinations. A high value of the VIF indicates a multicollinearity problem and the value higher than 10 is of concern (Armitage, 2001). We applied these criteria to select the variable with using the sample dataset which consisted of 196 subjects; 95 females and 101 males of from 30 to 69 years old with 49.3 mean, and their VFA are from 8.1 to $213.4 \mathrm{~cm}^{2}$ with 86.9 mean. The results are listed in Table 2 .

\begin{tabular}{|l|l|l|l|}
\hline No & Selected variables & VIF & AIC \\
\hline 1 & $a, b^{2}, \sqrt{a^{2}+b^{2}} Z_{S}, 1 / Z_{T}$ & 7.494421 & 1202.845 \\
\hline 2 & $a, b^{2}, a Z_{S}, 1 / Z_{T}$ & 8.583536 & 1202.953 \\
\hline 3 & $a, b^{2}, a b Z_{S}, 1 / Z_{T}$ & 9.680687 & 1203.16 \\
\hline 4 & $a, b^{2}, b Z_{S}, 1 / Z_{T}$ & 7.355794 & 1203.468 \\
\hline 5 & $a b, a^{2} Z_{S} 1 / Z_{T}$ & 5.527161 & 1204.678 \\
\hline 6 & $a b, a Z_{S}, 1 / Z_{T}$ & 4.653251 & 1205.513 \\
\hline 7 & $a^{2}, b^{2}, \sqrt{a^{2}+b^{2}} Z_{S}, 1 / Z_{T}$ & 7.767823 & 1205.883 \\
\hline 8 & $a^{2}, b^{2}, a Z_{S}, 1 / Z_{T}$ & 8.961434 & 1206.023 \\
\hline 9 & $a^{2}, b^{2}, b Z_{S}, 1 / Z_{T}$ & 7.451921 & 1206.098 \\
\hline 10 & $a, b^{2}, b Z_{S}, \sqrt{a^{2}+b^{2}} / Z_{T}$ & 8.578534 & 1206.461 \\
\hline
\end{tabular}

Table 2. Selected variables with VIF and AIC

\subsubsection{Experimental results and discussions}

We designed the experimental data set by using the three variables of gender, age, and waist circumference which is substituted to for VFA because it could not be used in the recruiting subjects phase. The number of subject is 180 (90 males +90 females) who were 22 to 80 years old (the mean is 49.95) and had from 65.8 to $120 \mathrm{~cm}$ of their waist circumferences (the mean 90.09). All of the subjects are measured by both the proposed method and a CT scan. 
The results were as follows; the correlation coefficient 0.88 , the errors mean -1.38 , and the errors standard deviation 27.28. The correlation coefficient between waist circumferences and CT scans is 0.77 .

As the results, visceral fat areas estimated by the proposed method achieved high correlation with ones by the CT scan. Waist circumference which is used in a medical checkup works well but the estimation accuracy is lower than the proposed method. Besides, it cannot differentiate VF from subcutaneous one and lean body in principle.

\section{Heart rate monitoring}

Among vital signals, a heart rate (HR) is an important index for understanding and diagnosing human's health condition. Especially, heart rate variability includes much information on health condition, for example, symptoms of cardiac disease, and conditions of autonomic nerve system (Kitney, 1980; Kobayashi, 1999). HR is measured in medical checkups and clinical diagnosis by electrocardiograph (ECG) as the gold standard. Besides the medical field, continuous monitoring of HRs during daily life activities is also strongly required because HR depends on activity intensity and monitoring HR might bring its information. Considering with usage in daily life, the monitoring should be realized without burden of human side. Regarding the burden, noninvasive, low intrusive, and unconscious sensing should be desired. In this section, an HR monitoring on bed by using an air pressure sensor (APS) is proposed for considering the unconscious and low cost biomedical sensing.

Because an APS is low cost and has high sensitivity, it could realize non invasive, non intrusive, and unconscious sensing on a bed with low cost. However, it brings too much information other than heart rate. The signal analysis such as filtering noise is required to realize its stable performance of HR estimation.

\subsection{Analysis domain}

There are two main analysis directions for measuring HRs from sensory signals; i.e., frequency domain analysis and time one. As frequency domain analysis, short term fast Fourier transformation (SFFT) is commonly used. SFFT is capable of monitoring global variability of target waveform. Because FFT assumes constant frequency, it does not extract microscopic variability. As time domain analysis, there have been several methods for HR measurement; i.e., peak detection, pattern matching, etc. Especially, pattern matching based on autocorrelation is commonly used to estimate HR variability from signals obtained via ECG monitor. Because of its capability of sensing HR variability, it is recommended for extraction and sensing of microscopic variability of HR. As HR monitoring in daily life requires the microscopic variability, time domain analysis is much more suitable for the usage.

\subsection{Causal analysis in biomedical sensing}

Regarding the transparency of biomedical sensing, causal analysis is a powerful tool since the causality is easy to be visualized, and makes the measurement principle clear.

There have been many practical studies on causal analysis. For instance, Thang et al. proposed a medical diagnosis support system based on oriental diagnosis knowledge (Thang, 2006). In their approach, the causality among some subject's symptoms and their diagnostic outcome is described by using RBF neural network. Nakajima et al. proposed a 
generic health management framework named Health Management Technology which is applied to not only human being but also manufacturing process, energy consumption management, and so forth (Nakajima, 2008a). Hata et al. suggested a concept named Human Health Care System of Systems which focus on health management, medical diagnosis, and surgical support (Hata, 2009). In the concept, the human health management technology is discussed from viewpoint of system of systems engineering. Marutschke et al. suggested that the causal analysis based on human-machine collaboration realizes transparent system model (Marutschke, 2009). From a viewpoint of theoretical development, lots of causal analysis theories have been proposed. Bayesian network describes statistical causality among phenomena observed from certain managed systems, and the statistical causality provides inference and reasoning functions (Pearl, 2001). Graphical model visualizes causality among components in complex systems (Miyagawa, 1991). Fuzzy logic helps intuitive representation of causality which is experts' implicit knowledge (Zadeh, 1996).

Through the discussions above, this section describes a transparent and accurate HR monitoring technology by employing an air pressure sensor and causal analysis among air pressure transit and HR.

\subsection{Measurement principle and system architecture}

\subsubsection{System design}

An HR monitoring equipment on a bed is implemented by using air pressure sensor (Hata, 2007). The equipment is not only capable of easy setup and application, but also unconscious and low intrusive. And the measurement principle is designed by employing causal analysis among air pressure and HR, and the cause-effect structure based on the designed causality is formed by using fuzzy logic (Zadeh, 1996; Tsuchiya, 2007; Tsuchiya, 2008).

Fig.9 shows the HR monitoring equipment. The human's body pressure is obtained via air pressure sensor, and the pressure is quantified into 1024 level (10bit) at $100 \mathrm{~Hz}$ by A/D converter. As a result, the HR transit is estimated from the quantified pressure. Fig.10 illustrates the principle of the measurement by using an air pressure sensor.

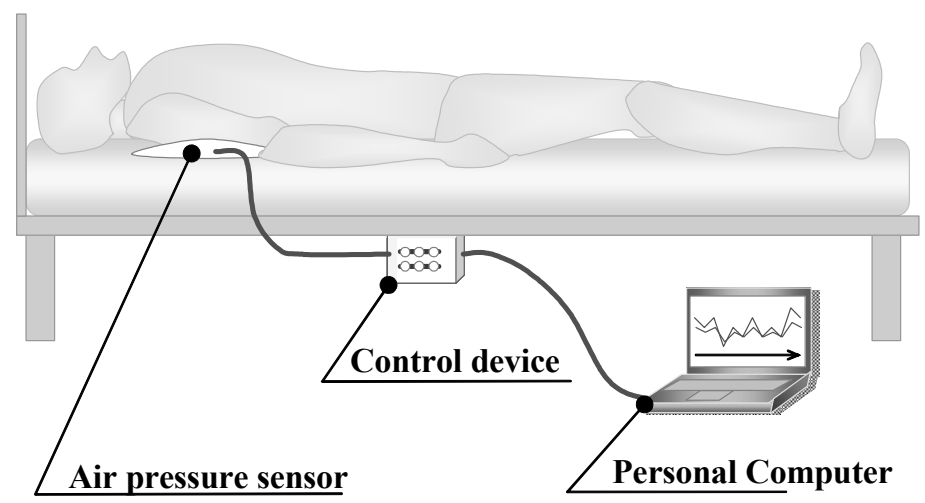

Fig. 9. Heart-rate monitoring equipment in sleep 

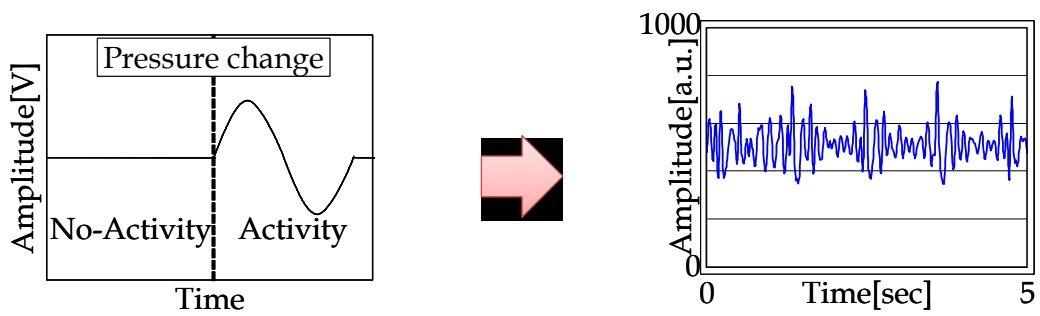

Fig. 10. Principle of the measurement by an air pressure sensor

\subsubsection{Heart rate estimation and causal analysis}

The basic idea of measuring HR monitoring is to extract heartbeats from pressure change of back in lying posture. The sensory signal superimposes not only heartbeat but also body movement and respiration. We need to extract the signal related to HR from the sensory source signal. In response to the requirement, causal analysis among air pressure and HR is employed to analysis and design the extraction method.

Firstly, the causality of heartbeat $H B$, body movement $B M V$, respiration $R S P$, and air pressure APS can be designed as the waveform analysis part as illustrated in Fig.11. Then, once $\mathrm{R}$ wave points $\tau_{R}$ could be extracted from $H B$ signal, $\mathrm{HR}$ variability could be calculated from R-R interval $\tau_{R R}$ which is the time differences of $\mathrm{R}$ waves in the same manner as ECG.

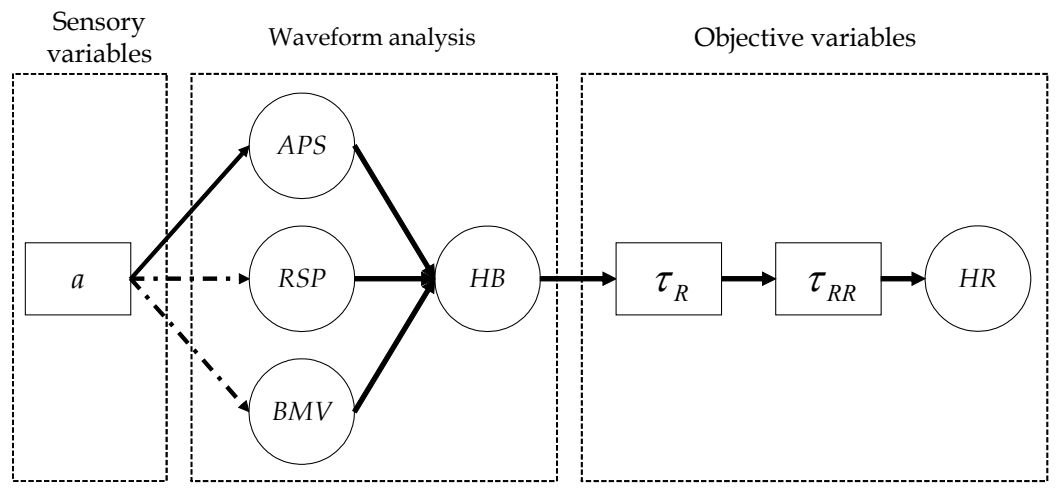

Fig. 11. Causality of heart rate estimation by using an air pressure sensor

As for $\tau_{R}$ extraction from pressure change, the pressure change involves not only heartbeat but also respiration and body movement. Because of the nature of the signals, it could be difficult to determine the precise position of R-waves $\tau_{R}$ by autocorrelation function and peak detection method. In this study, fuzzy logic is employed to formulate the knowledge about heartbeat.

Step 1. Firstly, full-wave rectification is applied to APS, and the pre-processed signal is determined as $x_{i}$.

Step 2. Then, the fuzzy logic based on the knowledge about $\tau_{R R}$ is applied to the preprocessed signal $x_{i}$. These fuzzy rules are described in the following. 
Knowledge 1 : The large pressure change is caused by heartbeat.

Knowledge 2 : Heartbeat interval does not change significantly.

According to the knowledge on heartbeat characteristics, the fuzzy rules are denoted in the following.

Rule 1 : IF $x_{i}$ is HIGH, THEN the degree of heartbeat point $\mu_{A m p}$ is HIGH.

Rule 2 : IF $t_{i}$ is CLOSE to $\bar{T}$, THEN the degree of heartbeat point $\mu_{\text {Int }}$ is HIGH.

where $\mu_{\text {Amp }}$ is the membership function of Rule $1, x_{i}$ is pre-processed pressure change, $t_{i}$ is the sampling point of obtained pressure change, $\bar{T}$ is the average of heartbeat intervals that calculated by using previous ten heartbeats, and $\mu_{I n t}$ is the membership function of Rule 2. Then, the membership functions respond to the fuzzy rules are illustrated in Fig.12(a) and 12(b), and formulae are equations (12)-(14) and (15), (16).

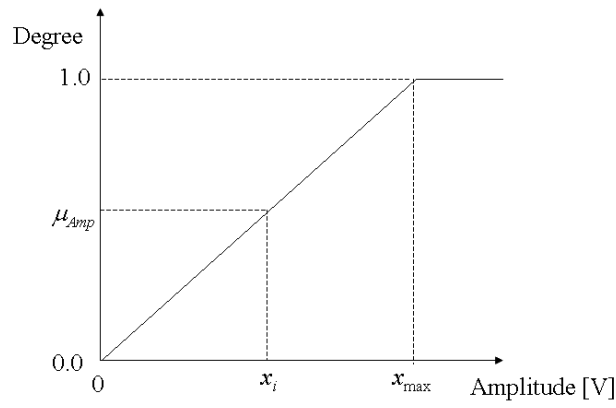

(a) Heartbeat amplitude

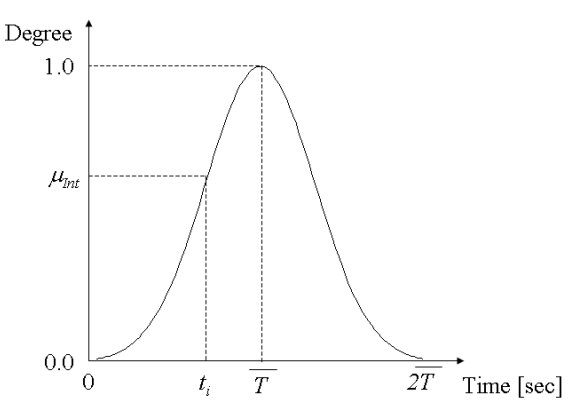

(b) Heartbeat interval

Fig. 12. Membership functions

$$
\begin{gathered}
\mu_{\text {Amp }}(i)= \begin{cases}0 & \text { if } x_{i}<x_{\min } \\
\frac{x_{i}-x_{\min }}{x_{\max }-x_{\min }} & \text { if } x_{\min } \leq x_{i} \leq x_{\max } \\
1 \quad & \text { if } x_{i}>x_{\max }\end{cases} \\
x_{\min }=\min \left(x_{\mathrm{APS}}\right) \\
x_{\max }=\max \left(x_{\mathrm{APS}}\right) \\
\mu_{\text {Int }}(i)=\exp \left(\frac{-\left(t_{i}-\bar{T}\right)^{2}}{2 \sigma^{2}}\right) \\
\sigma=\bar{T} / 3
\end{gathered}
$$


Step 3. Finally, $\mu_{i}$ is calculated by multiplying $\mu_{A m p}$ and $\mu_{\text {Int }}$ and the location with maximum $\mu_{i}$ is determined as heartbeat $H B$ as formulated in equation (17).

$$
\mu_{t}=\mu_{\mathrm{Amp}}(i) * \mu_{\mathrm{Int}}(i)
$$

\subsection{Experimental results}

In this experiment, the developed HR monitoring is compared with conventional and typical method that is based on autocorrelation functions. Table 3 shows the profile of each subject, and their correlations between HR changes obtained from the ECG and those obtained from the HR monitoring equipment. The results indicate that the proposed method achieved higher performance for all of the subjects. In particular, the correlation to ECG for the subject $\mathrm{A}$ and $\mathrm{E}$ is over 0.97 .

\begin{tabular}{l|l|l|l|l|l|l}
\hline \multirow{2}{*}{ Subject } & \multicolumn{4}{l|}{ Attribute } & \multicolumn{2}{l}{ Correlation coefficient } \\
\cline { 2 - 7 } & Age $[\mathrm{yrs}]$ & Gender & Height $[\mathrm{cm}]$ & Weight $[\mathrm{kg}]$ & Proposed & AC function \\
\hline \hline A & 23 & Male & $\mathbf{1 7 5}$ & 76 & 0.973 & 0.703 \\
\hline B & 23 & Male & 171 & 68 & 0.807 & 0.389 \\
\hline C & 23 & Male & 165 & 50 & 0.754 & 0.621 \\
\hline D & 25 & Male & 171 & 56 & 0.872 & 0.699 \\
\hline E & 22 & Male & 180 & 92 & 0.972 & 0.658 \\
\hline F & 22 & Male & 172 & 55 & 0.844 & 0.677 \\
\hline G & 23 & Male & 170 & 62 & 0.737 & 0.346 \\
\hline
\end{tabular}

Table 3. Experimental result on 7 males on a bed

Figure 13 shows an example of HR monitoring result for subject E. In the figure, the virtual axis is R-R interval (heartbeat interval), the horizontal axis is heartbeat count, the black line is R-R interval estimated by the proposed method, and the gray line is the one obtained by ECG. According the result around 200 beats, the proposed HR monitoring technology estimates the correct R-R intervals even if the significant change is occurred.

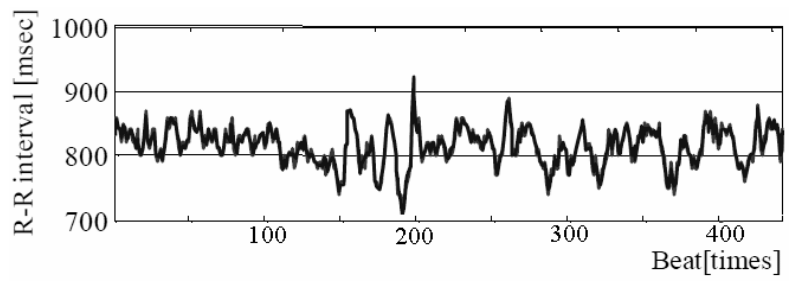

Fig. 13. An example of HR (R-R interval) monitoring result by using proposed method

\section{Summaries and conclusions}

In this chapter, the Smart Health Management Technology is proposed with introductions to its applications. The notion of the technology centers causality to realize the transparency and 
the cyclic evolution of a target and its management system. It consists of four functions; i.e., Measurement, Recognition, Estimation, and Evolution. Each function plays an important role for realizing quantification, classification, diagnosis and prognosis, and solution providing respectively. The reason why causality is employed as an essential representation is that causality can be readable and improvable via interaction between human and machine and their collaboration brings powerful and developmental solution. In the applications, the expected benefits were verified. In the visceral fat estimation, the causality was used for both measurement principle and its relationship with sensory variables. Firstly, the causality was designed by human experts. Then, variable selection and parameter tunings followed to derive the final estimation model. In the heart rate estimation, the design of causality was from the view points of wave analysis and objective variables. According to the wave analysis causality, fuzzy logic was employed for filtering sensory signal related to heart beat. The experiments were conducted to evaluate efficiency and effectiveness of the proposed method in the applications. The visceral fat estimation worked well as the correlation coefficient was 0.88 with X-ray CT scan. The heart rate estimation resulted that the mean of the correlation coefficient was 0.85 with electrocardiograph.

According to the notion of SHMT, Fig. 4 illustrates the causality from multivariate time series data. The applications introduced here provide mainly the measurement functionality. In the future, the accumulation of sensory data will realize the notion of diagnosis and prognosis based on causalities.

\section{References}

Akaike, H., (1974), A new look at the statistical model identification, IEEE Trans on Automatic Control, AC-19, No. 6, pp. 716-723 (1974)

Armitage, P., Berry, G., \& Matthews, J. N. S.,(2001), Statistical Methods in Medical Research, Wiley-Blackwell, 358-360 (2001)

Endo, M., Tsuruta, K., Kita, S., \& Nakajima, H., (2008), A Study of Cause-Effect Structure Acquisition for Anomaly Diagnosis in Discrete Manufacturing Process, Proceedings of 2008 IEEE International Conference on Systems, Man, and Cybernetics, 2099-2104

Examination Committee of Criteria for 'Obesit Disease' in Japan (ECCODJ), (2002), Japan Society for Study of Obesity, "New Criteria for 'obesity disease' in Japan," Cric J 66 (11), 987-992 (2002)

Gomi, T., (2005), Measurement of Visceral Fat/Subcutaneous Fat Ratio by 0.3 Tesla MRI, Radiation Medicine, 23 (8), 584-587 (2005)

Gopnik, A., and Schulz, L., (2007), Causal Learning - Psychology, Philosophy, and Computation, OXFORD University Press, ISBN 978-019517680, USA.

Hata, Y., Kamozaki, Y., Sawayama, T., Taniguchi, K. \& Nakajima, H., (2007), A heart pulse monitoring system by air pressure and ultrasonic sensor systems, Proceedings of IEEE System of Systems, 1-5 (2007).

Hata, Y., Kobashi, S. \& Nakajima, H, (2009), Human health care system of systems, IEEE System Journal, Vol.3, No. 2, pp.231-238 (2009).

Heckerman, D., Meek, C., \& Cooper, G., (1999), A Bayesian Approach to Causal Discovery, Chapter 4, Computation, Causation, and Discovery, AAAI PRESS/The MIT PRESS

Ho, K., Tsuchiya, N., Nakajima, H., Kuramoto, K., Kobashi, S., \& Hata, Y., Fuzzy Logic Approach to Respiration Detection by Air Pressure Sensor, Proceedings of 2009 IEEE International Conference on Fuzzy Systems, August, 911-915 (2009) 
Kitney, R.I. \& Rompelman, O., (1980), The Study of Heart Rate Variability, Clarendon Press, Oxford (1980).

Kobayashi, H., Ishibashi, K. \& Noguchi, H., (1999), Heart rate variability; an index for monitoring and analyzing human autonomic activities, AHS 18(2), 53-59 (1999).

Li, B.; Xu, Y. \& Choi, J. (1996). Applying Machine Learning Techniques, Proceedings of ASME 2010 4th International Conference on Energy Sustainability, pp. 14-17, ISBN 842-650823-3, Phoenix, Arizona, USA, May 17-22, 2010

Lucas, P., Games, J. A., \& Salmeron, A., (2007), Advances in Probabilistic Graphical Models, Springer,

Lukaski, H., Johnson, P., Bolonchuk, W., \& Lykken, G., (1985), Assessment of fat-free mass using bioelectrical impedance measurements of the human body, American Journal of Clinical Nutrition, 41, 810-817 (1985)

Marutschke, D. M., Nakajima, H., Tsuchiya, N., Yoneda, M., \& Iwami, T., (2008), CausalityBased Transparency And Accuracy In System Modeling with Human-Machine Collaboration, Proceedings of World Automation Congress 2008, pp. 1-6

Marutschke, D. M., Nakajima, H., Tsuchiya, N., Yoneda, M., \& Iwami, T. and Kamei, K., (2009), Actualization of Causality-Based Transparency and Accuracy in System Modeling with Human-Machine Collaboration, International Journal of Intelligent Computing in Medical Sciences and Image Processing, Vol.3, No. 2, pp. 131-141 (2009)

Messerli, F. H., \& Cotiga,D., (2005), Masked hypertension and white-coart hyptertension Therapic navigation between Scylla and Charybdis, Jouranal of the American College of Cardiology, Vol.46, No.3, pp.516-517, 2005.

Mittal, A., \& Kassim, A., (2007), Bayesian Network Technologies - Applications and Graphical Models, IGI Publishing,

Miyagawa, M., (1991), Statistical causal inference using graphical models, JJSS, Vol.29, No. 3, 327-356 (1991).

Nakajima, H., Hasegawa, Y., Tasaki, H., Iwami, T., \& Tuchiya, N. (2008a). Health Management Technology as a General Solution Framework, SICE Journal of Control, Measurement, and System Integration (SICE JCMSI), Vol.1, No.3, (May 2008), pp.257264, ISBN 978-4-339-89203-1

Nakajima, H., Hasegawa, Y., Tasaki, H., \& Kojitani, K., (2008b), SoS Aspects of Health Management Technology in Substrate Manufacturing Process, Proceedings of IEEE SMC System of Systems Engineering, 1-6, (2008)

Nakajima, H., Tasaki, H., Tsuchiya, N., Hamaguchi, T., \& Shiga, T., (2011), Visceral fat estimation method by bioelectrical impedance analysis and causal analysis, Proceedings of SPIE Defense, Security + Sensing, to appear (2011)

Pearl, J., (2000), Causality - Models, Reasoning, and Inference, Cambridge University Press, ISBN 978-0521773621, New York, USA.

Pearl, J., (2001), Models, Reasoning and Inference, Cambridge University Press, Cambridge (2001).

Ryo, M., Maeda, K., Onda, T., Katashima, M., Okumiya, A., Nishida, M., Yamaguchi, T., Funahashi, T., Matsuzawa, Y., Nakamura, T., \& Shimomura, I., (2005), A New Simple Method for the Measurement of Visceral Fat Accumulation by Bioelectrical Impedance, Diabetes Care, Vol. 28, No. 2, pp. 451-453 (2005)

Scharfetter, H., Schlager, T., Strollberger, R., Felsberger, R., Hutten, H., \& HinghoferSzalkay, H., (2001), Assessing abdominal fatness with local bioimpedance analysis: 
basics and experimental findings, International Journal of Obesity, Vol. 25, No. 4, $502-$ 511 (2001)

Scheines, R., Spirtes, P., Glymour, C., Meek, C., \& Richardson, T., (1999), Truth is among the Best Explanations : Finding Causal Explanations of Conditional Independence and Dependence, Chapter 5, Computation, Causation, and Discovery, AAAI PRESS/The MIT PRESS

Shiga, T., Oshima, Y., Kanai, H., Hirata, M., Hosoda, K., \& Nakao, K., A Simple Measurement Method of Visceral Fat Accumulation by Bioelectrical Impedance Analysis, Proceedings of ICEBI 2007, IFMBE 17, 687 - 690 (2007)

Shiga, T., Hamaguchi, T., Oshima, Y., Kanai, H., Hirata, M., Hosoda, K., \& Nakao, K., A new simple measurement system of visceral fat accumulation by bioelectrical impedance analysis, Proceedings of WC 2009 IFMBE 25/VII, pp. 338 - 341 (2009)

Spirtes, P., Glymour, C., \& Scheines, R., (2000), Causation, Prediction, and Search, second edition, The MIT Press,

Thang, C., Cooper, E.W. \& Hoshino, Y.,(2006), A proposed model of diagnosis and prescription in oriental medicine using RBF neural networks, JACIII 10(4), 458-464 (2006).

Tsuchiya, N., Yoneda, M., Nakajima, H., Hata, Y., Taniguchi, K., \& Sawayama, T., (2007), Fuzzy Extraction Method of Heart Rate by Air-Pressure Sensor," Proceedings of International Conference on Soft Computing and Human Sciences, 2-5 (2007)

Tsuchiya, N., Yamamoto, K., Nakajima, H., \& Hata, Y., (2008), A Comparative Study of Heart Rate Estimation via Air Pressure Sensor, Proceedings of 2008 IEEE International Conference on Systems, Man, and Cybernetics, 3077-3082 (2008)

Tsuchiya, N., Yoneda, M., \&Nakajima, H., (2010), Causal-effect structure transformation based on hierarchical representation for biomedical sensing, World Review of Science, Technology and Sustainable Development 2010, 7(1/2) (2010)

Whittaker, J., (1990), Graphical Models in Applied Multivariate Statistics, John Wiley \& Sons,

Wright, S., (1923), The method of path coefficients, Annuals Mathematical Statistics, 5, 161-215

Yamagoe, K. \& Togawa, T., "1.4 Perticularity of Bioinstrumentation and Sensing Method,"

Biomedical Sensors and Instruments, Corona Publishing Co.,Ltd. 2000 (In Japanese)

Yamaguchi, H., Nakajima, H., Taniguchi, K., Kobashi, S., Kondo, K., \& Hata, Y., (2007), Fuzzy Detection System of Behavior before Getting Out of Bed by Air Pressure and Ultrasonic Sensors, Proceedings of 2007 IEEE International Conference on Granular Computing, 114-119, (2007)

Yoneda, M., Tasaki, H., Tsuchiya, N., Nakajima, H., Hamaguchi, T., Oku, S., \& Shiga,T., (2007), A Study of Bioelectrical Impedance Analysis Methods for Practical Visceral Fat, Proceedings of 2007 IEEE International Conference on Granular Computing, pp. 622627, ISBN , Fremont, California, USA.

Yoneda, M., Tasaki, H., Tsuchiya, N., Nakajima, H., Hamaguchi, T., Oku, S., \& Shiga,T., (2008), Development of Visceral Fat Estimation Method based on Bioelectrical Impedance Analysis Method, Journal of SOFT, Vol. 20, No. 1, pp. 90-99, ISSN 13477986, Japan, (In Japanese).

Zadeh, L.A., (1996), Fuzzy Sets, Fuzzy Logic, Fuzzy System, World Scientific Publishing, ISBN 978-9810224226, Singapore. 


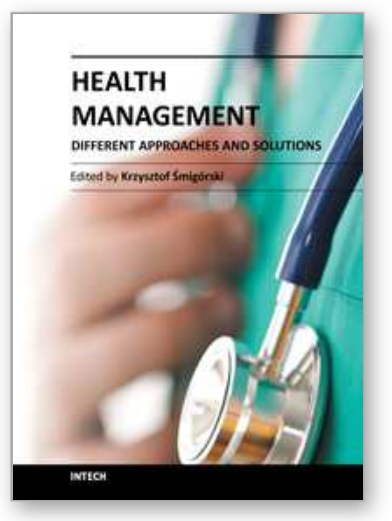

\author{
Health Management - Different Approaches and Solutions \\ Edited by Dr. Krzysztof Smigorski
}

ISBN 978-953-307-296-8

Hard cover, 498 pages

Publisher InTech

Published online 14, December, 2011

Published in print edition December, 2011

The development in our understanding of health management ensures unprecedented possibilities in terms of explaining the causes of diseases and effective treatment. However, increased capabilities create new issues. Both, researchers and clinicians, as well as managers of healthcare units face new challenges: increasing validity and reliability of clinical trials, effectively distributing medical products, managing hospitals and clinics flexibly, and managing treatment processes efficiently. The aim of this book is to present issues relating to health management in a way that would be satisfying for academicians and practitioners. It is designed to be a forum for the experts in the thematic area to exchange viewpoints, and to present health management's stateof-art as a scientific and professional domain.

\title{
How to reference
}

In order to correctly reference this scholarly work, feel free to copy and paste the following:

Hiroshi Nakajima (2011). Smart Health Management Technology, Health Management - Different Approaches and Solutions, Dr. Krzysztof Smigorski (Ed.), ISBN: 978-953-307-296-8, InTech, Available from: http://www.intechopen.com/books/health-management-different-approaches-and-solutions/smart-healthmanagement-technology

\section{INTECH}

open science | open minds

\section{InTech Europe}

University Campus STeP Ri Slavka Krautzeka 83/A 51000 Rijeka, Croatia Phone: +385 (51) 770447 Fax: +385 (51) 686166 www.intechopen.com

\section{InTech China}

Unit 405, Office Block, Hotel Equatorial Shanghai No.65, Yan An Road (West), Shanghai, 200040, China 中国上海市延安西路65号上海国际贵都大饭店办公楼405单元 Phone: $+86-21-62489820$

Fax: +86-21-62489821 
(C) 2011 The Author(s). Licensee IntechOpen. This is an open access article distributed under the terms of the Creative Commons Attribution 3.0 License, which permits unrestricted use, distribution, and reproduction in any medium, provided the original work is properly cited. 